\title{
Giant and severe pyoderma gangrenosum: about a
}

\section{case}

\begin{abstract}
Pyoderma gangrenosum is a non infectious neutrophilic dermatosis with unknown origin. In contrast to its name, PG is neither an infectious nor gangrenous condition. Half of the cases have associated systemic diseases. ${ }^{1}$ Here in, we report a case of a 46-year-old male with no significant history of malignancies, systemic vasculitis, arthritis, inflammatory bowel disease, fungal infection, drugs, or cutaneous injury was referred to our Department for multiple ulcers appears on the trunk and limbs evolving with relapses and remissions. Clinical examination showed diffusely distributed, two, well-defined, deep purulent ulcers on the back and the calf measuring 4 to $40 \mathrm{~cm}$, with severe oozing and erosions. Laboratory tests revealed a white cell count of $10.23 \times 109 / 1$, hemoglobin $120 \mathrm{~g} / 1$, and C-reactive protein $12 \mathrm{mg} / \mathrm{l}$. All other tests were normal, including hepatic function tests, renal tests, antinuclear antibodies, anti-neutrophil cytoplasmic antibodies, HIV, hepatitis, and Venereal Disease Research Laboratory test for syphilis. A body scan and colonoscopy were performed and was normal. The patient was treated with systemic glucocorticoids (60mg daily) and topical wound care with partial improvement.
\end{abstract}

Keywords: dermatosis, malignancies, vasculitis, arthritis
Volume 4 Issue 6 - 2020

\author{
S El Kadiri, Z Douhi, R Chaoui, S Elloudi, H \\ Baybay, FZ Mernissi \\ Department of Dermatology, CHU Hassan II , Fez, Morocco
}

Correspondence: El Kadiri Salma, Department of Dermatology, Morocco, Email elkadiris@hotmail.com

Received: December 21, 2020 | Published: December 29 2020

\section{Introduction}

Pyoderma gangrenosum (PG) is a noninfectious neutrophilic dermatosis with unknown origin. In contrast to its name, $P G$ is neither an infectious nor gangrenous condition. Half of the cases have associated systemic diseases. ${ }^{1}$ Here in, we report a case of a rare and giant PG.

\section{Case report}

A-46-year-old male with no significant history of malignancies, systemic vasculitis, arthritis, inflammatory bowel disease, fungal infection, drugs, or cutaneous injury was referred to our department for multiple ulcers appears on the trunk and limbs. These ulcers progressed by relapses and remissions giving way to cribriform scars for the 3 past years. At this moment, the patient was in a penitentiary institution for 3 years.

In the beginning, the patient presented painful pustules on his abdomen. They evolved quickly into blisters and ulcerations. They progressed into large ulcerations in the back. At that time, the patient had surgical debridement with worsening of his condition. Dermatological examination revealed two well-defined, deep purulent ulcers on the back measuring $40 * 20 \mathrm{cms}$ (Figure 1) and an ulceration on the posterior calf with oozing (Figure 3). Cribriform scars from previous lesions involved approximately $60 \%$ of the body surface (Figure 2). Biopsy specimens demonstrated the findings of an ulcer with heavy neutrophil infiltration of the dermis without giant cells of leukocytoclastic vasculitis. Cutaneous cultures for bacteria, fungus, and mycobacteria were negative. The histology and tissue culture findings were compatible with a pyodermagangrenosum. Laboratory tests revealed a white cell count of $10.24 \times 109 / 1$, hemoglobin $120 \mathrm{~g} / 1$, and C-reactive protein $12 \mathrm{mg} / \mathrm{l}$. All other tests were normal, including hepatic function tests, renal tests, HIV, immulogic tests (antinuclear antibodies, anti-neutrophil cytoplasmic antibodies), hepatitis, and Venereal Disease Research Laboratory test for syphilis. A body scan and colonoscopy were performed and was normal. They didn't reveal also extracutaneous organ inflammation. The patient was treated with systemic glucocorticoids (60mg daily) and topical wound care. After 2 weeks, a partial healing of the ulcer was noted. The ulcer of the back was completely healed after one month of treatment (Figure 4). But, he presented a recurrence in the chest (Figure 5). Thereafter the patient became lost to follow-up.

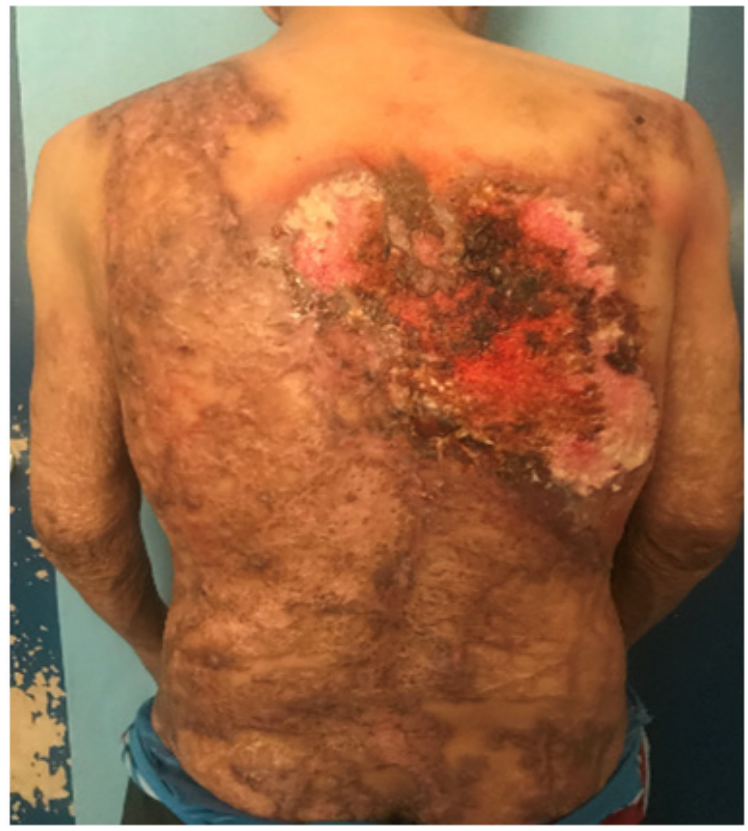

Figure I Pyodermagangrneosum on the back. 


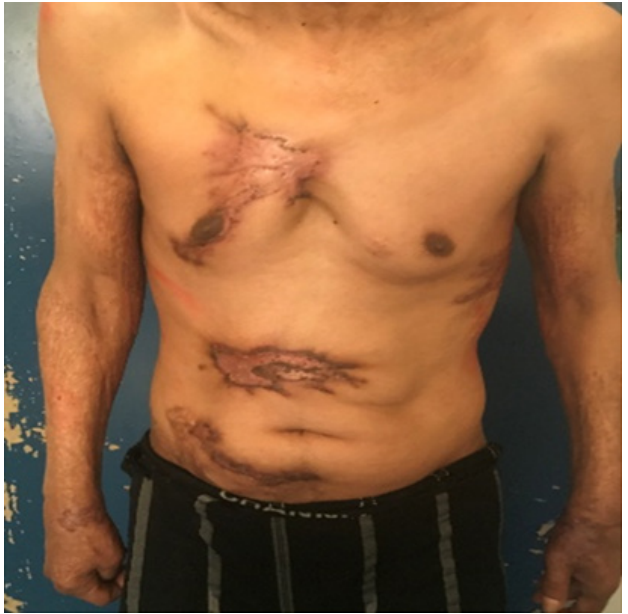

Figure 2 Cribriform and atrophic scar from pyoderma gangrenosum.

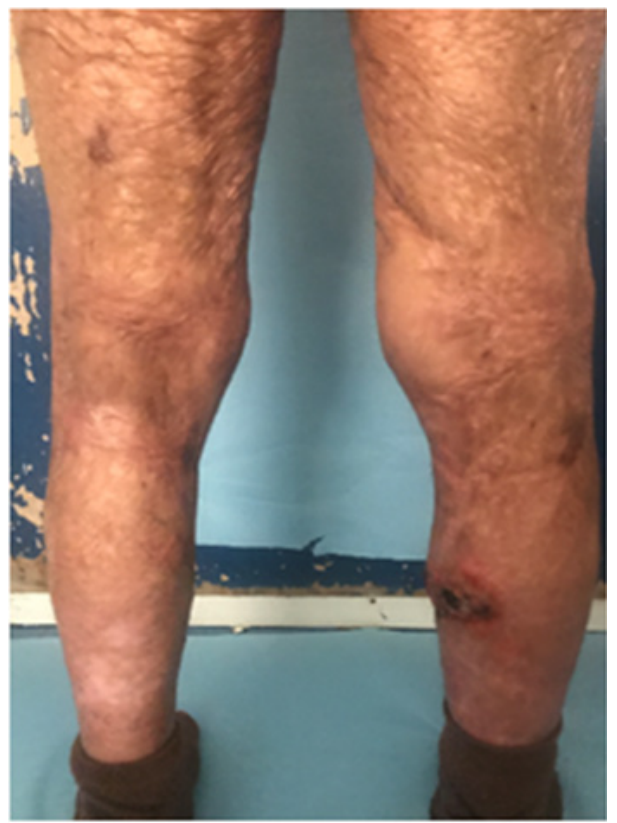

Figure 3 Pyoderma gangrenosum on the calf.

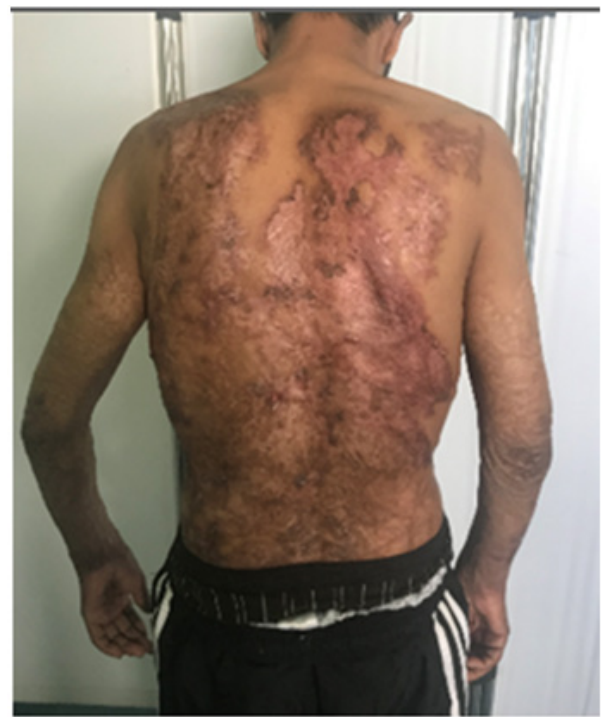

Figure 4 Complete healing of the pyoderma gangrenosum.

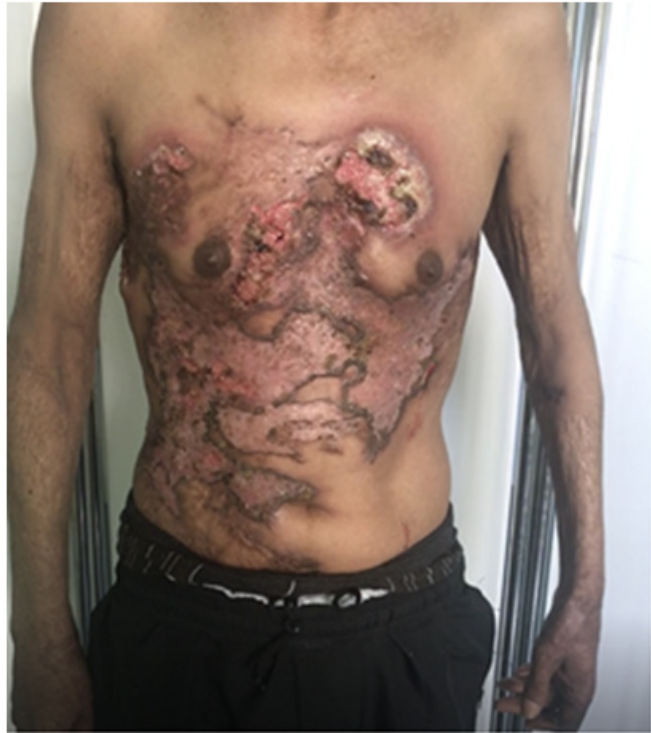

Figure 5 Active pyodermagangrneosum on the with cribriformscar (control).

\section{Discussion}

PG is an ulcerative disorder of unknown origin. Brunsting is credited with the first description of $\mathrm{PG}$ in $1930 .{ }^{1} \mathrm{PG}$ is a rare disease that affects people of all ages, most commonly in the $40 \mathrm{~s}$ to $60 \mathrm{~s}$, with a female predominance and rare pediatric cases. In half of the cases with PG, patients have an associated underlying medical condition or trigger, particularly inflammatory bowel disease, inflammatory arthritis, and hematologic disorders (myelodysplastic syndrome, leukemia, immunoglobulin A paraproteinemia, others). ${ }^{2}$ They are also drug-induced cases but they remain rare. There are five clinical subtypes, with ulcerative the most common; others include bullous, vegetative, pustular, and peristomal. Typical PG presents first as an inflammatory papule and pustule that rapidly and painfully progresses to an ulcer or erosion with tissue necroses and enlargement of the area. The ulcer is surrounded by a violaceous border with surrounding erythema. ${ }^{3} 30 \%$ of lesions occur via pathergy corresponding to the abnormal development of skin lesions at the sites of injury including minor injuries, surgical scars, and burns. These pathergy induced PG are often misdiagnosed as cellulitis. ${ }^{4,5}$ In this case, the diagnosis of PG was delayed. Despite broad-spectrum antibiotics and surgical debridement, the lesions worsened over the years. There is no gold standard in pyoderma treatment. It should include the skin lesion and the underlying disease. For limited disease, topical and intralesional therapy can control disease without systemic medications. While corticosteroids are used in diffuse or recalcitrant disease. Cyclosporine and TNF inhibitors have been used in many reports with variable response. New targeted therapies, including anti-IL-6 and anti-IL-17 agents, have the potential for future studies. ${ }^{3}$ Although the history of surgical debridement, we performed complete investigations to rule out other illnesses or associated diseases.

\section{Conclusion}

To the best of our knowledge, we report a rare PG in a patient with a historical presentation. We couldn't find a similar report in the literature been reported before. Although the history of surgical debridement, we believe that where there is smoke, there is fire. Monitoring overtime for internal malignancies, inflammatory bowel disease, and inflammatory arthritis should have required in our patient but unfortunately, the follow up was lost. 


\section{Conflicts of interest}

There are no conflicts of interest.

\section{Acknowledgments}

None.

\section{Funding}

None.

\section{References}

1. Brunsting LA, Goeckerman WH, O’Leary PA. Pyoderma (echthyma) gangrenosum: clinical and experimental observations in five cases occurring in adults. Arch Dermatol Syphilol. 1930;22:655-680.
2. Niu R, Zheng J, Ding $\mathrm{D}$, et al. Giant pyoderma gangrenosum in a patient with ulcerative colitis: A case report. Medicine (Baltimore). 2020;99(6):e18795

3. Jiang YY, Li J, Li Y, et al. Comparison of clinical features between pyoderma gangrenosum concomitant by inflammatory bowel disease and idiopathic pyoderma gangrenosum. Chin Med J (Engl). 2017;130:26742679 .

4. Patel DK, et al. Pyoderma gangrenosum with pathergy. J Plast ReconstrAesthet Surg. 2017;70(7):884-892.

5. Weizman AV, Huang B, Targan S, et al. Pyodermagangrenosum among patients with inflammatory bowel disease: a descriptive cohort study. $J$ Cutan Med Surg. 2014;18(5):361. 